\title{
Caracterização e Seleção de Clones de Capim-Elefante (Pennisetum purpureum Schum.) na Zona da Mata de Pernambuco ${ }^{1}$
}

\author{
Alexandre Carneiro Leão de Mello², Mário de Andrade Lira ${ }^{3}$, José Carlos Batista \\ Dubeux Júnior ${ }^{4}$, Mércia Virgínia Ferreira dos Santos ${ }^{5}$, Erinaldo Viana de Freitas ${ }^{6}$
}

RESUMO - O estudo visou caracterizar uma coleção de 71 clones de capim-elefante e selecionar os mais promissores para serem testados sob pastejo. A caracterização foi realizada na Estação Experimental do Cedro/IPA, no município de Vitória de Sto. Antão/PE, nas estações chuvosa e seca. Cada clone foi representado por parcelas não repetidas de $10 \mathrm{~m}^{2}$. Segundo análise de correlação linear, os clones mais produtivos em relação à MS, também apresentaram maiores alturas de planta e de meristemas apicais, diâmetros de colmo, relações colmo/folha $(\mathrm{C} / \mathrm{F})$, números de perfilhos basais e total e comprimentos de folha. Excetuando-se a relação $\mathrm{C} / \mathrm{F}$, as mesmas características também correlacionaram-se positivamente com a produção de lâmina foliar (LF). Foi observada uma correlação linear positiva e altamente significativa $(r=0,9517)$ entre as produções de MS e de LF. Baseando-se nos materiais que apresentaram as maiores produções de LF/área, foram selecionados 10 clones: Mineirão/IPEACO, Hexaplóide, Mole de Volta Grande, King Grass, 591-76 ou Cameroon, CE 5 A.D., Gigante de Pinda, CE 4 A.D., Elefante da Colômbia e Vrukwona.

Palavras-chave: avaliação, forrageiras, melhoramento genético, produtividade

\section{Characterization and Selection of ElephantGrass Clones (Pennisetum purpureum Schum.) at the Pernambuco Forest Zone}

\begin{abstract}
This work aimed to characterize an elephantgrass collection (Pennisetum purpureum Schum.) comprising 71 clones and selecting the best ones to be tested later under grazing conditions. It was carried out at Cedro Experimental Station - IPA, located in Vitória de Santo Antão, forest zone of Pernambuco State. The period of evaluation included both the raining and the dry season. Each clone was represented by a non-replicated plot of $10 \mathrm{~m}^{2}$. According to linear correlation analysis, the more productive clones also showed highest plant height and highest growing point height, greatest stem diameter, highest stem/leaf ratio, highest basal and total tiller numbers and greatest leaf lenght. The same characteristics above were also positively correlated with the leaf blade yield, except stem/leaf ratio. There was a high correlation coefficient $(r=0.9517)$ between dry matter yield and leaf blade yield. The selection of clones to be tested under grazing conditions was based on the materials that showed the highest leaf blade yield. These materials were: Mineirão/IPEACO, Hexaplóide, Mole de Volta Grande, King Grass, 591-76 or Cameroon, CE 5 AD, Gigante de Pinda, CE 4 AD, Elefante da Colômbia and Vrukwona.
\end{abstract}

Key Words: evaluation, forage plant, plant breeding, productivity

\section{Introdução}

O Nordeste brasileiro apresenta atualmente $14 \%$ do rebanho bovino do país, o que representa cerca de 22 milhões de cabeças (Anuário da Pecuária Brasileira, 1998). Entretanto, a área de pastagens existente não é suficiente para atender esse rebanho, havendo uma tendência de déficit na alimentação desses animais.

O capim-elefante, apesar de tradicionalmente ser utilizado na forma de capineira, tem mostrado excelente desempenho quando utilizado sob pastejo, pro- piciando ótimos resultados tanto para a produção de carne como para a produção de leite. Entretanto, a maioria das informações encontradas sobre essa gramínea referem-se ao seu uso sob a forma de capineira. O desenvolvimento de programas de melhoramento, visando a obtenção de cultivares que apresentem características desejáveis para a utilização sob pastejo, constitui uma importante demanda do setor pecuário.

Assim, esse trabalho objetivou caracterizar uma coleção de 71 clones de capim-elefante em duas

\footnotetext{
${ }_{1}^{1}$ Parte da Dissertação de Mestrado em Produção Animal/UFRPE do primeiro autor.

${ }^{2}$ Aluno de Doutorado/USP-ESALQ, bolsista da FAPESP. Av. 17 de agosto, 9/704, Parnamirim, Recife/PE; CEP: 52060-590.

E.mail:aclmello@esalq.usp.br

3 Pesquisador do IPA, bolsista do CNPq. E.mail: mlira@hotlink.com.br

${ }^{4}$ Prof. Depto. de Zootecnia/UFRPE. Bolsista do CNPq. E.mail: dubeux@netpe.com.br

${ }^{5}$ Profa. Depto. de Zootecnia/UFRPE. E.mail: rmsantos@elogica.com.br

${ }^{6}$ Pesquisador do IPA.
} 
estações do ano, visando, posteriormente, selecionar os acessos mais adaptados e produtivos a serem testados para formação de pastagem na região da Zona da Mata de Pernambuco.

\section{Material e Métodos}

A coleção de 71 clones de capim-elefante (Tabela 1) foi instalada na Estação Experimental do Cedro, município de Vitória de Sto. Antão/PE, pertencente a Empresa Pernambucana de Pesquisa Agropecuária-IPA. A Estação apresenta como coordenadas geográficas $08^{\circ} 08^{\prime} 00^{\prime}$ S de latitude e $35^{\circ} 22^{\prime} 00^{\prime}$ " W Gr. de longitude, precipitação pluviométrica anual de $1.025 \mathrm{~mm}$, com maior con- centração entre os meses de março e julho, umidade relativa do ar média de $67 \%$ e temperatura média de $25,4^{\circ} \mathrm{C}$. O tipo climático foi definido como $\mathrm{C}_{1} \mathrm{~S}_{2} \mathrm{~A}^{\prime}$ a (seco sub-úmido megatérmico) e o tipo de solo podzólico vermelho amarelo (PVA) equivalente eutrófico, com topografia predominantemente ondulada (Encarnação, 1980; IPA, 1994). O solo onde foi implantada a coleção foi classificado como franco-arenoso e apresentou as seguintes características: $\mathrm{pH}=6,50 ; \mathrm{Ca}^{++}=2,20 \mathrm{meq} / 100 \mathrm{~g}$ de solo; $\mathrm{P}=76,47 \mathrm{ppm} ; \mathrm{Mg}^{++}=1,05 \mathrm{meq} / 100 \mathrm{~g}$ de solo; $\mathrm{K}^{+}=0,20\left(\mathrm{meq} / 100 \mathrm{~g}\right.$ de solo; $\mathrm{Al}^{+++}=0,00 \mathrm{meq} /$ $100 \mathrm{~g}$ de solo; $\mathrm{Na}^{+}=0,17 \mathrm{meq} / 100 \mathrm{~g}$ de solo; $\mathrm{H}^{+}=0,66 \mathrm{meq} / 100 \mathrm{~g}$ de solo; $\mathrm{N}=0,07 \%$ e matéria orgânica $1,01 \%$.

Tabela 1 - Coleção de clones de capim-elefante, Vitória de Sto. Antão/PE

Table 1 - Elephantgrass clones collection, Vitória de Sto. Antão/PE

\begin{tabular}{|c|c|c|c|}
\hline $\begin{array}{l}\mathrm{N}^{0} \text { de ordem } \\
\text { Order number }\end{array}$ & $\begin{array}{l}\text { Clone } \\
\text { Clone }\end{array}$ & $\begin{array}{l}\mathrm{N}^{\mathrm{O}} \text { de ordem } \\
\text { Order number }\end{array}$ & $\begin{array}{l}\text { Clone } \\
\text { Clone }\end{array}$ \\
\hline 1 & CE 1 A.D ${ }^{1}$ & 37 & 414-76(Elef. da Colômbia) ${ }^{4}$ \\
\hline 2 & CE 2 A.D ${ }^{1}$ & 38 & 475-76 (Mole de V.Grande) 4 \\
\hline 3 & CE 3 A. $\mathrm{D}^{1}$ & 39 & 481-76 (Duro de V.Grande) 4 \\
\hline 4 & CE 4 A.D ${ }^{1}$ & 40 & 479-76 ou Terezópolis 4 \\
\hline 5 & CE 5 A.D ${ }^{1}$ & 41 & 903-77 ou Australiano ${ }^{7}$ \\
\hline 6 & CE 6 A.D ${ }^{1}$ & 42 & $587-76$ ou Itapemirim ${ }^{7}$ \\
\hline 7 & CE 7 A. $\mathrm{D}^{1}$ & 43 & $590-76$ ou IAC ${ }^{8}$ \\
\hline 8 & CE 8 A.D ${ }^{1}$ & 44 & Três Rios ${ }^{7}$ \\
\hline 9 & CE 9 A.D ${ }^{1}$ & 45 & Gigante de pinda $^{7}$ \\
\hline 10 & CE 10 A.D ${ }^{1}$ & 46 & Porto Rico - 534-B ${ }^{7}$ \\
\hline 11 & CE 11 A.D ${ }^{1}$ & 47 & P-241 Piracicaba ${ }^{7}$ \\
\hline 12 & CE 12 A.D ${ }^{1}$ & 48 & Kizozi $^{7}$ \\
\hline 13 & CE 13 A.D ${ }^{1}$ & 49 & Mott-F17 \\
\hline 14 & Vrukuona A.D $\mathrm{D}^{1}$ & 50 & Guaçu/IZ ${ }^{7}$ \\
\hline 15 & Venezuela A.D ${ }^{1}$ & 51 & Cuba $115^{7}$ \\
\hline 16 & Cameroon A.D ${ }^{1}$ & 52 & Cuba $116^{7}$ \\
\hline 17 & 474-76 ou Mineiro ${ }^{2}$ & 53 & Cuba $169^{7}$ \\
\hline 18 & 464-76 ou Costa Rica ${ }^{2}$ & 54 & King Grass ${ }^{7}$ \\
\hline 19 & 483-76 ou Turrialba ${ }^{2}$ & 55 & Roxo de Botucatu 7 \\
\hline 20 & $591-76$ ou Cameroon ${ }^{3}$ & 56 & Mineirão/IPEACO ${ }^{7}$ \\
\hline 21 & 586-76 ou Vrukuona ${ }^{3}$ & 57 & Vrukwona $^{7}$ \\
\hline 22 & $589-76$ ou Taiwan A-146 4 & 58 & Cameroon $^{7}$ \\
\hline 23 & $452-76$ ou Taiwan A- $148^{4}$ & 59 & $\mathrm{CPAC}^{7}$ \\
\hline 24 & $469-76$ ou Taiwan A-1444 & 60 & Napiezinho $^{7}$ \\
\hline 25 & $454-76$ ou Taiwan A- $25^{4}$ & 61 & IJ-7125 cv. Empasc $308^{7}$ \\
\hline 26 & 472-76 ou Pusa Napier n. $1^{4}$ & 62 & IJ-7127 cv. Empasc $310^{7}$ \\
\hline 27 & 419-76 ou Pusa Napier n. $2^{4}$ & 63 & IJ-7127 cv. Empasc $309^{7}$ \\
\hline 28 & 415-76 ou Mercker 5 & 64 & IJ-7136 \\
\hline 29 & $418-76$ (Mercker Sta Rita) $^{6}$ & 65 & IJ-71399 \\
\hline 30 & 415-76 ou $\mathrm{SEA}^{4}$ & 66 & IJ-714 ${ }^{9}$ \\
\hline 31 & 468-76 ou Mercker México 4 & 67 & Goiano $^{7}$ \\
\hline 32 & 478-76 ou Merc ${ }^{4}$ & 68 & $\mathrm{CAC} 262^{7}$ \\
\hline 33 & $470-76$ ou Napier SEA ${ }^{4}$ & 69 & Ibitinema $^{7}$ \\
\hline 34 & $477-76$ ou Napier ${ }^{7}$ & 70 & BAG-50 7 \\
\hline 35 & 421-76 ou Napier Goiano ${ }^{4}$ & 71 & Hexaplóide $^{7}$ \\
\hline 36 & 473-76 ou Elef. de Pinda ${ }^{4}$ & & \\
\hline
\end{tabular}

Procedências: ${ }^{1} \mathrm{IRI} ;{ }^{2}$ Sete Lagoas; ${ }^{3} \mathrm{ESALQ} ;{ }^{4}$ IPEACS; ${ }^{5}$ Não disponível.; ${ }^{6} \mathrm{IPEACO} ;{ }^{7} \mathrm{CNPGL} ;{ }^{8} \mathrm{IAC} ;{ }^{9} \mathrm{EMPASC}$. 
Cada clone foi plantado em duas linhas de 5 metros de comprimento, espaçadas de 1 metro, em parcelas não repetidas. Na instalação da coleção foi realizada uma adubação com $30 \mathrm{~kg} / \mathrm{ha}$ de $\mathrm{N}, 90 \mathrm{~kg} / \mathrm{ha}$ de $\mathrm{P}_{2} \mathrm{O}_{5}$ e $60 \mathrm{~kg} / \mathrm{ha}$ de $\mathrm{K}_{2} \mathrm{O}$.

Foram realizadas avaliações em toda a coleção nas épocas seca e chuvosa. A época chuvosa foi subdividida nos anos de 1997 (fevereiro/março) e 1998 (abril/maio), onde parte das características foi avaliada no primeiro ano e as restantes no segundo ano. A avaliação na época seca foi realizada nos meses de setembro e outubro de 1997.

$\mathrm{Na}$ estação chuvosa foi utilizada irrigação nos períodos em que não houve precipitação. Na época chuvosa de 1997, a precipitação total ocorrida para o período que compreendeu a avaliação foi de $218,0 \mathrm{~mm}$ e na de 1998, de 87,0 mm. Na estação seca, ocorreram precipitações atípicas para o período, totalizando 34,8 mm de chuvas.

Após a completa implantação da coleção, foi realizado em 21/01/97 um corte de uniformização a $30 \mathrm{~cm}$ do solo. Imediatamente após o corte, foi realizada uma adubação nitrogenada com o equivalente a $100 \mathrm{~kg} / \mathrm{ha}$ de $\mathrm{N}$.

As avaliações de altura de planta e perfilhamento foram realizadas aos 56 dias de crescimento das plantas após o corte em cada estação. Para altura de planta foram tomadas ao acaso quatro medições/ parcela, desde o solo até as extremidades das folhas apicais completamente expandidas, sendo obtido o valor médio das quatro medições realizadas. O número de perfilhos foi observado separadamente em perfilhos axilares e basais, contados em um mesmo metro linear/parcela. Estes parâmetros foram avaliados nas estações seca e chuvosa de 1997.

Para o parâmetro florescimento, foram observadas e anotadas as datas de início e término do mesmo, bem como a duração em dias. O início foi considerado quando aproximadamente 5\% dos colmos se encontravam em antese e o término quando aproximadamente $95 \%$ ultrapassavam este estádio.

As demais características foram avaliadas quando a coleção se apresentava com 60 dias de rebrota, após o corte de uniformização.

O diâmetro de colmo foi medido a $80 \mathrm{~cm}$ do solo com o auxílio de paquímetro, em cinco colmos escolhidos aleatoriamente dentro da parcela, sendo anotado o valor médio das medições realizadas.

A avaliação de relação colmo/folha foi realizada em cinco perfilhos aleatórios, que apresentavam brotação basilar como origem. A relação foi obtida pela separação manual das lâminas foliares e colmos e determinação do peso seco $\left(\mathrm{a} 65^{\circ} \mathrm{C}\right)$ de cada componente. Foi considerada largura da folha, a distância entre as duas extremidades laterais da parte mediana da folha e o comprimento, a distância entre a inserção e a parte apical da folha, sem esticá-la. Nos dois parâmetros foram observadas as folhas do terço médio das plantas, quando foram tomadas quatro medidas ao acaso para cada parâmetro e calculadas as médias das observações.

A altura dos meristemas apicais foi avaliada em quatro colmos que apresentavam a brotação basilar como origem, tomados aleatoriamente, onde os mesmos eram cortados rente ao solo e abertos, com a ajuda de uma faca, determinando assim a altura entre o solo e meristema apical, sendo anotados os valores médios das quatro observações. Foram considerados ângulos foliares, o ângulo formado entre a parte central (o colmo) e a inserção da folha, sendo o ângulo medial tomado no terço médio e o ângulo apical no terço superior das plantas, com o auxílio de um transferidor. Foram tomadas três medições/parcela e obtida a média.

A produção de matéria seca foi avaliada nas duas estações, tendo-se obtido como amostra a forragem colhida em um corte a $30 \mathrm{~cm}$ do solo, no mesmo local utilizado para as avaliações do número de perfilhos. A determinação da produção de lâminas foliares foi realizada apenas na estação seca. Para esta determinação, foram utilizados os dados de produção de MS e de relação $\mathrm{C} / \mathrm{F}$, seguindo a seguinte metodologia:

$$
\begin{gathered}
\text { I) } \mathrm{C} / \mathrm{F}=\mathrm{R} \rightarrow \mathrm{C}=\mathrm{R} \text {. F e II) } \mathrm{F}+\mathrm{C}=\mathrm{MS} \rightarrow \\
\mathrm{F}=\mathrm{MS}-\mathrm{C}
\end{gathered}
$$

em que: $\mathrm{F}=$ folhas $\mathrm{C}=$ colmos; $\mathrm{R}=$ relação $\mathrm{C} / \mathrm{F}$ e $\mathrm{MS}=$ produção de matéria seca.

Substituindo I em II:

$$
\begin{gathered}
\mathrm{F}=\mathrm{MS}-(\mathrm{R} . \mathrm{F}) \rightarrow \mathrm{F}+\mathrm{R} . \mathrm{F}=\mathrm{MS} \rightarrow \\
\mathrm{F}=\mathrm{MS} / \mathrm{R}+1
\end{gathered}
$$

Por se tratar de um trabalho de caracterização de uma coleção de clones de capim-elefante com parcelas não repetidas, não foi realizada análise de variância para os caracteres estudados, sendo os dados analisados para as duas épocas do ano. Entretanto, foram obtidas análises de regressão linear simples, para todas as características em relação às produções de matéria seca e de lâmina foliar. Além disso, foi determinado o intervalo de confiança para a média a um nível de confiança de $95 \%$ de probabilidade, e os coeficientes de variação para todas as características avaliadas. 


\section{Resultados e Discussão}

Altura de planta, altura de meristemas apicais (AMA), perfilhamento e florescimento

Na Tabela 2 são observados os valores de alturas de planta e de meristemas apicais dos cinco clones que apresentaram maiores e menores valores, média geral, intervalo de confiança para a média e o coeficiente de variação para toda a coleção.

O clone BAG-50 apresentou a maior altura da coleção tanto na estação chuvosa como na seca, com 300,0 e $242,5 \mathrm{~cm}$, respectivamente. Já os clones CE 6 A.D., com 225,0 cm e o Três Rios, com 121,2 cm, apresentaram as menores alturas nas estações chuvosa e seca, respectivamente.

Foi observada uma relação positiva entre a produção de matéria seca (PMS) e a altura de planta nas duas estações do ano (Seca: $\mathrm{PMS}=-5,11+0,05 . \mathrm{h} ; \mathrm{R}^{2}=$ 0,19; Chuvosa: $\left.\mathrm{PMS}=-30,16+0,15 \cdot \mathrm{h} ; \mathrm{R}^{2}=0,20 ; \mathrm{P}<0,01\right)$. Estes dados concordam com os de Oliveira (1988) e Santos et al. (1994), que comprovaram que os clones mais produtivos também apresentaram maior altura. Os dados de altura de planta encontrados nesse trabalho, indicam que, independentemente da estação do ano, a seleção de plantas de capim-elefante baseadas na PMS deve estar relacionada às plantas de porte mais alto. A altura também relacionou-se positivamente com a produção de lâmina foliar por área na estação seca (PLF)(PLF=-1,79+0,02.h; $\left.\mathrm{R}^{2}=0,15 ; \mathrm{P}<0,01\right)$, indicando que plantas mais altas e produtivas tendem a apresentar maior produção de folhas, característica forrageira das mais importantes em capim-elefante para corte e/ou pastejo (Lira et al., 1998).

Observou-se ampla variação da AMA entre os clones, sendo a média dos dez clones superiores foi de $133,4 \mathrm{~cm}$, enquanto para os dez inferiores, de $63,5 \mathrm{~cm}$, na estação chuvosa. Nesta estação, o clone que apresentou o maior valor para AMA foi o IJ-7127 cv. Empasc 310, com 152,2 cm, e na seca foi o BAG-50, com 101,7 cm, estando este bem acima dos demais clones nesta estação. Os materiais que apresentaram os menores valores para esse caracter foram o 469-76 ou Taiwan A-144, com 58,5 cm e o Três Rios, com $32,2 \mathrm{~cm}$, para as estações chuvosa e seca, respectivamente.

A AMA também relacionou-se positivamente com a PMS nas duas estações do ano (Seca: PMS = 1,03+0,06.AMA; $\mathrm{R}^{2}=0,15$; Chuvosa: $\mathrm{PMS}=$ $-4,32+0,16$.AMA; $\left.\mathrm{R}^{2}=0,42 ; \mathrm{P}<0,01\right)$ indicando novamente, com base nas condições deste estudo, que plantas com elevadas AMA e, conseqüentemente, elevadas alturas de planta podem ser selecionadas, objetivando-se altas PMS. Exemplificando, os dois clones com maiores AMA, Empasc-310 e o BAG-50, também apresentaram as mais altas PMS da coleção na estação chuvosa.

Os valores de perfilhamento axilar variaram de 0 a 44 (chuvosa) e 0 a 36 (seca) e de basais de 15 a 56 (chuvosa) e 19 a 73 (seca). Estes valores (Tabela 3) podem ser considerados baixos, quando comparados aos relatados por Botrel et al. (1998), que encontraram uma média de 151 e 35 perfilhos $/ \mathrm{m}^{2}$, para perfilhos aéreos e basais, respectivamente. Entretanto, quando comparados aos resultados de Lira et al. (1998), os valores de número de perfilhos encontram-se acima dos obtidos por estes autores, que relataram para sete clones de capim-elefante, uma média de 24 e 38 perfilhos $/ \mathrm{m}^{2}$, para basais e axilares, respectivamente.

Corsi, citado por Gomide (1997), estudando o perfilhamento de Panicum maximum, cita que o peso dos perfilhos tem maior correlação com a produção de matéria seca do que a quantidade de perfilhos, o que corrobora a relação positiva dos perfilhos basais com a PMS na estação seca (PMS=1,12+0,1.PB; $\mathrm{R}^{2}$ $=0,19: \mathrm{P}<0,01)$, visto que os perfilhos basais são maiores e mais pesados. Isto vem demonstrar a importância do perfilhamento basal na produtividade do capim-elefante na estação seca, indicando que para áreas com baixos índices de pluviosidade, podese optar por clones de capim-elefante com altas brotações de perfilhos basais, objetivando-se satisfatória PMS e PLF.

Dos 71 clones da coleção apenas 34 floresceram, apresentando grande variabilidade tanto no período de início e término, como também no período de duração. Os clones mais precoces, foram: CE 5 A.D., 477-76 ou Napier (CNPGL), 421-76 ou Napier Goiano, 479-76 ou Terezópolis e Mineirão/IPEACO, os quais ultrapassaram $5 \%$ da antese 56 dias após o corte. Dos clones que floresceram, os mais tardios foram: CE 11 A.D. e 419-76 ou Pusa Napier n. 2, os quais levaram 129 dias após o corte de uniformização para ultrapassar os $5 \%$ da antese.

Não foi observado o florescimento de nenhum clone na estação seca. Isto pode ser explicado devido à mudança do fotoperíodo nesta estação, onde o período de luminosidade aumenta, com a redução do período escuro e, segundo Delvin (1997), o período de escuro é o maior responsável pela floração das plantas de dias curtos. 
Tabela 2 - Altura de planta e altura do meristema apical de clones de capim-elefante Table 2 - Plant height and growth point height of elephantgrass clones

\begin{tabular}{|c|c|c|c|}
\hline \multicolumn{4}{|c|}{$\begin{array}{l}\text { Altura de planta }(\mathrm{cm}) \\
\quad \text { Plant height }(\mathrm{cm})\end{array}$} \\
\hline \multicolumn{2}{|c|}{$\begin{array}{c}\text { Estação chuvosa } \\
\text { Rainy season }\end{array}$} & \multicolumn{2}{|c|}{$\begin{array}{l}\text { Estação seca } \\
\text { Dry season }\end{array}$} \\
\hline Clones de menor altura & Altura $(\mathrm{cm})$ & Clones de menor altura & Altura $(\mathrm{cm})$ \\
\hline Clones with the lowest height & Height $(\mathrm{cm})$ & Clones with the lowest height & Height $(\mathrm{cm})$ \\
\hline CE 6 A.D & 225,0 & Três Rios & 121,2 \\
\hline Três Rios & 232,5 & CPAC & 140,0 \\
\hline Kizozi & 238,7 & CE 1 A.D & 155,0 \\
\hline CPAC & 243,7 & Kizozi & 156,2 \\
\hline 469-76 ou Taiwan A-144 & 245,0 & CE 12 A.D & 160,0 \\
\hline Clones de menor altura & Altura $(\mathrm{cm})$ & Clones de menor altura & Altura $(\mathrm{cm})$ \\
\hline Clones with the lowest height & Height $(\mathrm{cm})$ & Clones with the lowest height & Height $(\mathrm{cm})$ \\
\hline 477-76(Napier/CNPGL) & 292,5 & Cuba 169 & 221,2 \\
\hline Porto Rico - 534 - B & 292,5 & King Grass & 223,7 \\
\hline Cuba 169 & 295,0 & 472-76 ou Pusa Napier n.1 & 226,2 \\
\hline Mott-F1 & 300,0 & Mott-F1 & 236,2 \\
\hline BAG-50 & 300,0 & BAG-50 & 242,5 \\
\hline $\mathrm{IC}_{(\mathrm{m}) 0,95}{ }^{3}$ & $268,1 \pm 3,79$ & $\mathrm{IC}_{(\mathrm{m}) 0,95^{3}}$ & $186,7 \pm 4,88$ \\
\hline$C I_{(m) 0.95}$ & & $\mathrm{CI}_{(\mathrm{m}) 0.95}$ & \\
\hline $\mathrm{CV}(\%)^{4}$ & 6,1 & $\mathrm{CV}(\%)^{4}$ & 11,2 \\
\hline \multicolumn{4}{|c|}{$\begin{array}{l}\text { Altura do meristema apical (AMA), cm } \\
\text { Growth point height }(G P H), \mathrm{cm}\end{array}$} \\
\hline \multicolumn{2}{|c|}{$\begin{array}{l}\text { Estação chuvosa }{ }^{1} \\
\quad \text { Rainy season }\end{array}$} & \multicolumn{2}{|c|}{$\begin{array}{l}\text { Estação seca }{ }^{1} \\
\text { Dry season }\end{array}$} \\
\hline Clones de menor AMA & $\mathrm{AMA}(\mathrm{cm})$ & Clones de menor AMA & $\mathrm{AMA}(\mathrm{cm})$ \\
\hline Clones with the lowest GPH & $G P H(\mathrm{~cm})$ & Clones with the lowest GPH & $G P H(\mathrm{~cm})$ \\
\hline 469-76 ou Taiwan A-144 & 58,5 & Três Rios & 32,2 \\
\hline CE 8 A.D & 61,5 & IJ-7141 & 38,0 \\
\hline Vrukwona & 61,5 & CE 1 A.D & 39,5 \\
\hline CPAC & 61,5 & Guaçu/IZ & 39,7 \\
\hline Cameroon & 63,2 & Kizozi & 40,2 \\
\hline Clones de menor AMA & $\mathrm{AMA}(\mathrm{cm})$ & Clones de menor AMA & $\operatorname{AMA}(\mathrm{cm})$ \\
\hline Clones with the lowest GPH & $G P H(\mathrm{~cm})$ & Clones with the lowest GPH & $G P H(\mathrm{~cm})$ \\
\hline 589-76 ou Taiwan A-146 & 132,2 & 419-76 ou Pusa Napier n.2 & 87,5 \\
\hline 468-76(Mercker México) & 135,2 & King Grass & 88,7 \\
\hline Gigante de pinda & 139,7 & CE 5 A.D & 90,7 \\
\hline BAG-50 & 147,0 & Mott-F1 & 92,5 \\
\hline IJ-7127 cv. Empasc 310 & 152,2 & BAG-50 & 101,7 \\
\hline $\mathrm{IC}_{(\mathrm{m}) 0,95^{3}}$ & $93,3 \pm 5,34$ & $\mathrm{IC}_{(\mathrm{m}) 0,95^{3}}$ & $62,8 \pm 3,70$ \\
\hline $\mathrm{CI}_{(\mathrm{m}) 0.95}$ & & $\mathrm{CI}_{(\mathrm{m}) 0.95}$ & \\
\hline $\mathrm{CV}(\%)$ & 24,6 & $\mathrm{CV}(\%)^{4}$ & 25,3 \\
\hline
\end{tabular}

${ }_{1}^{1}$ Avaliação realizada em 1997.

2 Avaliação realizada em 1998.

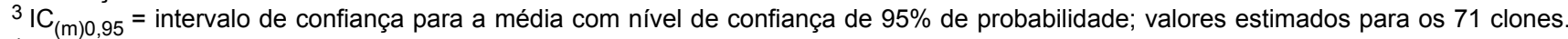

${ }^{4}$ Coeficiente de variação.

1 Evaluation performed in 1997.

2 Evaluation performed in 1998.

${ }^{3} \mathrm{Cl}_{(\mathrm{m}) 0.95}=$ Mean confidence interval (confidence level of 95\%); values obtained from 71 clones.

${ }^{4}$ Coefficient of variation. 
Tabela 3 - Número de perfilhos basais e axilares de clones de capim-elefante

Table 3 - Number of basal and axillary tillers of elephantgrass clones

\begin{tabular}{|c|c|c|c|}
\hline \multicolumn{4}{|c|}{$\begin{array}{l}\text { Número de perfilhos basais (NPB) } \\
\text { Number of basal tillers (NBT) }\end{array}$} \\
\hline \multicolumn{2}{|c|}{$\begin{array}{l}\text { Estação chuvosa } \\
\text { Rainy season }\end{array}$} & \multicolumn{2}{|c|}{$\begin{array}{l}\text { Estação seca }{ }^{1} \\
\text { Dry season }\end{array}$} \\
\hline Clones de menor NPB/m & $\mathrm{NPB} / \mathrm{m}$ & Clones de menor NPB/m & $\mathrm{NPB} / \mathrm{m}$ \\
\hline Clones with the lowest $N B T / m$ & $N B T / m$ & Clones with the lowest $N B T / m$ & $N B T / m$ \\
\hline IJ-7141 & 15 & IJ-7141 & 19 \\
\hline 478-76 ou Merc & 17 & 464-76 ou Costa Rica & 21 \\
\hline 473-76 ou Elef. de Pinda & 18 & IJ-7127 cv. Empasc 310 & 24 \\
\hline 468-76(Mercker México) & 20 & Ibitinema & 24 \\
\hline 464-76 ou Costa Rica & 21 & Venezuela A.D & 26 \\
\hline Clones de maior NPB/m & $\mathrm{NPB} / \mathrm{m}$ & Clones de maior NPB/m & $\mathrm{NPB} / \mathrm{m}$ \\
\hline Clones with the highest NBT/m & $N B T / m$ & Clones with the highest NBT/m & $N B T / m$ \\
\hline CE 1 A.D & 45 & Hexaplóide & 60 \\
\hline 475-76 ou Mole V. Grande & 48 & 481-76 (Duro de V. Grande) & 62 \\
\hline $590-76$ ou IAC & 50 & CE 1 A.D & 63 \\
\hline Três Rios & 50 & 475-76 (Mole de V. Grande) & 65 \\
\hline 903-77 ou Australiano & 56 & $590-76$ ou IAC & 73 \\
\hline $\begin{array}{c}\mathrm{IC}_{(\mathrm{m}) 0,95} \\
C I_{(m) 0.95}\end{array}$ & $32,2 \pm 1,79$ & $\begin{array}{c}\mathrm{IC}_{(\mathrm{m}) 0,95} \\
C I_{(m) 0.95}\end{array}$ & $37,9 \pm 2,58$ \\
\hline $\mathrm{CV}(\%)^{3}$ & 23,9 & $\mathrm{CV}(\%)^{3}$ & 29,3 \\
\hline \multicolumn{4}{|c|}{$\begin{array}{c}\text { Número de perfihos axilares (NPA) } \\
\text { Number of axillary tillers (NAT) }\end{array}$} \\
\hline \multicolumn{2}{|c|}{$\begin{array}{c}\text { Estação chuvosa }^{1} \\
\text { Rainy season }\end{array}$} & \multicolumn{2}{|c|}{$\begin{array}{l}\text { Estação seca }{ }^{1} \\
\text { Dry season }\end{array}$} \\
\hline Clones de menor NPA/m & $\mathrm{NPA} / \mathrm{m}$ & Clones de menor NPA/m & $\mathrm{NPA} / \mathrm{m}$ \\
\hline Clones with the lowest NAT/m & $N A T / m$ & Clones with the lowest NAT/m & $N A T / m$ \\
\hline Cuba 115 & 0 & CE 5 A.D & 0 \\
\hline 469-76 ou Taiwan A-144 & 1 & CE 10 A.D & 0 \\
\hline Venezuela A.D & 2 & Venezuela A.D & 0 \\
\hline Cuba 116 & 2 & 483-76 ou Turrialba & 0 \\
\hline Cameroon & 2 & 586-76 ou Vrukuona & 0 \\
\hline Clones de maior NPA/m & $\mathrm{NPA} / \mathrm{m}$ & Clones de maior NPA/m & $\mathrm{NPA} / \mathrm{m}$ \\
\hline Clones with the highest NAT/m & $N A T / m$ & Clones with the highest NAT/m & $N A T / m$ \\
\hline CE 13 A.D & 29 & 415-76 ou Mercker & 18 \\
\hline IJ-7141 & 30 & CE 13 A.D & 21 \\
\hline Três Rios & 31 & IJ-7136 & 26 \\
\hline 477-76(Napier/CNPGL) & 34 & Goiano & 27 \\
\hline 468-76(Mercker México) & 44 & Três Rios & 36 \\
\hline $\begin{array}{c}\mathrm{IC}_{(\mathrm{m}) 0,95} \\
C I_{(m) 0.95}\end{array}$ & $13,3 \pm 2,02$ & $\begin{array}{l}\mathrm{IC}_{(\mathrm{m}) 0,95}{ }^{2} \\
C I_{(m) 0.95}\end{array}$ & $7,2 \pm 1,60$ \\
\hline $\mathrm{CV}(\%)^{3}$ & 65,4 & $\mathrm{CV}(\%)^{3}$ & 95,8 \\
\hline \multicolumn{4}{|c|}{$\begin{array}{l}1 \text { Avaliação realizada em } 1997 . \\
2 \mathrm{IC}_{(\mathrm{m}) 0,95}=\text { intervalo de confiança para a média com um nível de confiança de } 95 \% \\
{ }^{3} \text { Coeficiente de variação. } \\
1 \text { Evaluation performed in } 1997 . \\
\left.2 \mathrm{Cl}_{(\mathrm{m}) 0.95}=\text { mean confidence interval (confidence level of } 95 \%\right) ; \text { values obtained from } 71 \text { clones. } \\
3 \text { Coefficient of variation. }\end{array}$} \\
\hline
\end{tabular}


O florescimento tardio é mais desejável para a produção do capim-elefante tanto sob corte como para pastejo, visto que a partir do momento em que o processo de floração é iniciado, os meristemas apicais cessam a produção de novas folhas para, a partir daí ocorrer a diferenciação e formação da inflorescência, tendo com isso uma interrupção na produção de folhas e de biomassa.

Diâmetro de colmo (Ø) e Relação colmo/folha (C/F)

Os clones 473-76 ou Elefante de Pinda, CE 12 A.D. e o 419-76 ou Pusa Napier n. 2 destacaram-se dos demais por apresentar maior diâmetro de colmo na estação chuvosa (Tabela 4), estando o restante dos clones na faixa da média geral. Na estação seca, os clones com maiores diâmetros de colmo foram Cuba 116, Cuba 115 e o Mineirão/IPEACO (Tabela 4), o que pode indicar maior resistência desses materiais à seca, uma vez que os mesmos mantiveram praticamente os mesmos valores nas duas estações. Os clones Três Rios e CPAG não apresentaram colmos a altura de $80 \mathrm{~cm}$ na estação seca, indicando o baixo desenvolvimento desses materiais nas condições adversas de umidade.

Os dados de diâmetro de colmo encontrados estão de acordo com a amplitude de variação encontrada em capim-elefante por Xavier et al. (1995), que relataram valores entre 15 e $19 \mathrm{~mm}$, porém foram superiores, quando comparados aos valores citados por Lira et al. (1998), também tomados a $80 \mathrm{~cm}$ do solo e que apresentaram média para sete clones de $9,62 \mathrm{~mm}$.

Foram observadas relações positivas entre o diâmetro de colmo e a PMS e a PLF na estação seca (PMS=1,88+0,3.Ø; $\mathrm{R}^{2}=0,15 ; \mathrm{PLF}=1,37+0,13 . \varnothing ;$ $\left.\mathrm{R}^{2}=0,12 ; \mathrm{P}<0,01\right)$. Esses resultados sugerem que, objetivando-se maior produtividade de matéria seca e de folhas na estação seca, podem-se selecionar clones de colmos de maior diâmetro.

$\mathrm{Na}$ estação chuvosa, a média dos dez clones com maiores relações $\mathrm{C} / \mathrm{F}$ foi de 6,0 , a média dos dez clones com menores relações $\mathrm{C} / \mathrm{F}$ foi de 1,4 e a média geral situou-se em 3,3. Já na estação seca, a média geral situou-se em 0,8 , a média dos dez superiores em 1,3 e a dos dez inferiores em 0,4. Esses dados sugerem que o menor crescimento das plantas na estação seca é determinado por um menor alongamento dos colmos e consequentemente uma média de relação $\mathrm{C} / \mathrm{F}$ mais baixa para esta estação.
Os clones CE 4 A.D., com C/F de 6,8 e o 483-76 ou Turrialba, com C/F de 6,7, apresentaram os maiores valores deste caráter na estação chuvosa, enquanto o clone 415-76 ou SEA, com 0,8 (Tabela 4), apresentou o menor valor, merecendo destaque, visto que apesar do maior alongamento do colmo nesta estação, este clone foi capaz de produzir uma considerável quantidade de folhas, reduzindo assim sua relação $\mathrm{C} / \mathrm{F}$, o que seria mais desejável. Na estação seca, o clone CE 8 A.D., com $\mathrm{C} / \mathrm{F}=1,7$ e o clone 59176 ou Cameroon, com $\mathrm{C} / \mathrm{F}=0,2$, foram os que apresentaram a maior e a menor relação do caráter, respectivamente, destacando-se o segundo, que apesar do baixo alongamento do colmo registrado nesta estação, este clone apresentou uma produção de folhas satisfatória, reduzindo assim, sua relação $\mathrm{C} / \mathrm{F}$.

A relação $\mathrm{C} / \mathrm{F}$ assume grande importância na utilização do capim-elefante sob pastejo, visto que com baixos valores tem-se maior proporção de folhas e, conseqüentemente, melhor qualidade de forragem, permitindo-se assim maior seletividade do animal na sua dieta.

A relação $\mathrm{C} / \mathrm{F}$ apresentou relação positiva com a $\mathrm{PMS}$ na estação seca $\left(\mathrm{PMS}=2,59+2.95 . \mathrm{C} / \mathrm{F} ; \mathrm{R}^{2}=0,10\right.$; $\mathrm{P}<0,01)$. Este resultado concorda com os obtidos por Gonçalves et al. (1979) e Botrel et al. (1994), indicando tendência de que materiais mais produtivos em termos de PMS também apresentem maiores relações $\mathrm{C} / \mathrm{F}$.

Largura de folhas (LF) e comprimento de folhas (CF)

Os clones apresentaram LF média de 3,7 e 2,9 $\mathrm{cm}$, para as estações chuvosa e seca, respectivamente, destacando-se o clone 469-76 ou Taiwan A-144, na época chuvosa e os clones Cuba 169 e Venezuela A.D., na estação seca (Tabela 5). O CF médio foi de 78,2 e 71,4 cm, para as estações chuvosa e seca, respectivamente, tendo se destacado os clones 470-76 ou Napier SEA e 414-76 ou Elefante da Colômbia na estação chuvosa, e o 473-76 ou Elefante de Pinda, na estação seca.

Não foram observadas regressões lineares entre a LF e PMS, como também com a PLF. Já o CF apresentou relação positiva com a PMS $\left(\mathrm{PMS}=-7,04+0,17 . \mathrm{CF} ; \mathrm{R}^{2}=0,21 ; \mathrm{P}<0,01\right)$ e com a PLF (PLF $\left.=-3,61+0,09 . C F ; R^{2}=0,23 ; \mathrm{P}<0,01\right)$ na estação seca, demonstrando haver importância do comprimento das folhas de capim-elefante sobre a produtividade, principalmente em plantas destinadas 
Tabela 4 - Relação colmo/folha e diâmetro de colmo de clones de capim-elefante Table 4 - Stem/leaf ratio and stem diameter of elephantgrass clones

\begin{tabular}{|c|c|c|c|}
\hline \multicolumn{4}{|c|}{$\begin{array}{l}\text { Relação colmo/folha }(\mathrm{C} / \mathrm{F}) \\
\text { Stem/leafratio }(\mathrm{S} / \mathrm{L})\end{array}$} \\
\hline \multicolumn{2}{|c|}{$\begin{array}{l}\text { Estação chuvosa }^{1} \\
\text { Rainy season }\end{array}$} & \multicolumn{2}{|c|}{$\begin{array}{l}\text { Estação seca }{ }^{1} \\
\text { Dry season }\end{array}$} \\
\hline Clones de menor $\mathrm{C} / \mathrm{F}$ & $\mathrm{C} / \mathrm{F}$ & Clones de menor $\mathrm{C} / \mathrm{F}$ & $\mathrm{C} / \mathrm{F}$ \\
\hline Clones with the lowest $S / L$ & $S / L$ & Clones with the lowest $S / L$ & $S / L$ \\
\hline 415-76 ou SEA & 0,8 & 591-76 ou Cameroon & 0,2 \\
\hline IJ-7139 & 1,0 & 468-76 ou Mercker México & 0,3 \\
\hline $590-76$ ou IAC & 1,3 & 473-76 (Elefante de Pinda) & 0,3 \\
\hline 470-76 ou Napier SEA & 1,4 & Três Rios & 0,3 \\
\hline Cuba 169 & 1,4 & Guaçu/IZ & 0,3 \\
\hline Clones de maior $\mathrm{C} / \mathrm{F}$ & $\mathrm{C} / \mathrm{F}$ & Clones de maior $\mathrm{C} / \mathrm{F}$ & $\mathrm{C} / \mathrm{F}$ \\
\hline Clones with the highest $S / L$ & $S / L$ & Clones with the highest $S / L$ & $S / L$ \\
\hline Kizozi & 6,2 & BAG-50 & 1,2 \\
\hline Cuba 115 & 6,2 & CE 3 A.D & 1,3 \\
\hline King Grass & 6,3 & Goiano & 1,3 \\
\hline 483-76 ou Turrialba & 6,7 & 454-76 ou Taiwan A-25 & 1,4 \\
\hline CE 4 A.D & 6,8 & CE 8 A.D & 1,7 \\
\hline $\mathrm{IC}_{(\mathrm{m}) 0,95^{2}}$ & $3,3 \pm 0,35$ & $\mathrm{IC}_{(\mathrm{m}) 0,95^{2}}$ & $0,8 \pm 0,07$ \\
\hline $\mathrm{CI}_{(\mathrm{m}) 0.95}$ & & $C I_{(m) 0.95}$ & \\
\hline $\operatorname{CV}(\%)^{3}$ & 45,4 & $\mathrm{CV}(\%)^{3}$ & 37,5 \\
\hline \multicolumn{4}{|c|}{$\begin{array}{l}\text { Diâmetro de colmo }(\mathrm{DC}), \mathrm{mm} \\
\text { Stem diameter }(\mathrm{SD}), \mathrm{mm}\end{array}$} \\
\hline \multicolumn{2}{|c|}{$\begin{array}{l}\text { Estação chuvosa }{ }^{1} \\
\text { Rainy season }\end{array}$} & \multicolumn{2}{|c|}{$\begin{array}{l}\text { Estação seca }{ }^{1} \\
\text { Dry season }\end{array}$} \\
\hline Clones de menor DC & $\mathrm{DC}(\mathrm{mm})$ & Clones de menor DC & $\mathrm{DC}(\mathrm{mm})$ \\
\hline Clones with the lowest $S D$ & $S D(m m)$ & Clones with the lowest SD & $S D(\mathrm{~mm})$ \\
\hline 479-76 ou Terezópolis & 9,8 & Três Rios & 0,0 \\
\hline 415-76 ou Mercker & 10,4 & CPAC & 0,0 \\
\hline Kizozi & 10,8 & Guaçu/IZ & 4,5 \\
\hline Napiezinho & 10,8 & Cameroon A.D & 5,0 \\
\hline 478-76 ou Merc & 11,4 & 415-76 ou SEA & 6,0 \\
\hline Clones de maior DC & $\mathrm{DC}(\mathrm{mm})$ & Clones de maior DC & $\mathrm{DC}(\mathrm{mm})$ \\
\hline Clones with the highest SD & $S D(m m)$ & Clones with the highest $S D$ & $S D(m m)$ \\
\hline Cuba 116 & 17,0 & BAG-50 & 15,2 \\
\hline Goiano & 17,0 & King Grass & 16,2 \\
\hline 419-76 ou Pusa Napier n.2 & 18,4 & Mineirão/IPEACO & 16,2 \\
\hline CE 12 A.D & 19,5 & Cuba 115 & 16,5 \\
\hline 473-76 (Elefante de Pinda) & 20,6 & Cuba 116 & 18,5 \\
\hline $\begin{array}{c}\mathrm{IC}_{(\mathrm{m}) 0,95}{ }^{2} \\
\mathrm{CI}_{(m) 0.95}\end{array}$ & $14,4 \pm 0,46$ & $\begin{array}{l}\mathrm{IC}_{(\mathrm{m}) 0,95^{2}} \\
C I_{(m) 0.95}\end{array}$ & $10,4 \pm 0,79$ \\
\hline $\mathrm{CV}(\%)^{3}$ & 13,9 & $\mathrm{CV}(\%)^{3}$ & 32,7 \\
\hline
\end{tabular}

\footnotetext{
${ }^{1}$ Avaliação realizada em 1997.
}

$2 \mathrm{IC}_{(\mathrm{m}) 0,95}=$ intervalo de confiança para a média com um nível de confiança de $95 \%$ de probabilidade; valores estimados para os 71 clones.

${ }^{3}$ Coeficiente de variação.

${ }^{1}$ Evaluation performed in 1997.

${ }^{2} \mathrm{Cl}_{(\mathrm{m}) 0.95}=$ Mean confidence interval (confidence level of 95\%); values obtained from 71 clones.

${ }^{3}$ Coefficient of variation. 
Tabela 5 - Largura de folhas e comprimento de folhas de clones de capim-elefante

Table 5 - Leaf width and leaf lenght of elephantgrass clones

\begin{tabular}{|c|c|c|c|}
\hline \multicolumn{4}{|c|}{$\begin{array}{l}\text { Largura de folhas (LF), cm } \\
\text { Leaves widht }(L W), \mathrm{cm}\end{array}$} \\
\hline \multicolumn{2}{|c|}{$\begin{array}{l}\text { Estação chuvosa }^{1} \\
\text { Rainy season }\end{array}$} & \multicolumn{2}{|c|}{$\begin{array}{c}\text { Estação seca }{ }^{1} \\
\text { Dry season }\end{array}$} \\
\hline Clones de menor LF & $\mathrm{LF}(\mathrm{cm})$ & Clones de menor LF & $\mathrm{LF}(\mathrm{cm})$ \\
\hline Clones with the lowest $L W$ & $L W(\mathrm{~cm})$ & Clones with the lowest $L W$ & $L W(\mathrm{~cm})$ \\
\hline Ibitinema & 2,3 & Kizozi & 2,1 \\
\hline BAG-50 & 2,6 & Ibitinema & 2,1 \\
\hline CPAC & 2,7 & 479-76 ou Terezópolis & 2,2 \\
\hline CE 3 A.D & 2,8 & Três Rios & 2,2 \\
\hline CE 6 A.D & 2,9 & CE 7 A.D & 2,3 \\
\hline Clones de maior LF & $\mathrm{LF}(\mathrm{cm})$ & Clones de maior LF & $\mathrm{LF}(\mathrm{cm})$ \\
\hline Clones with the highest $L W$ & $L W(\mathrm{~cm})$ & Clones with the highest $L W$ & $L W(\mathrm{~cm})$ \\
\hline Hexaplóide & 4,6 & Cameroon A.D & 3,8 \\
\hline 591-76 ou Cameroon & 4,8 & 468-76 ou Mercker México & 3,8 \\
\hline Vrukwona & 4,8 & 473-76 (Elefante de Pinda) & 3,9 \\
\hline P-241 Piracicaba & 4,9 & Venezuela A.D & 4,0 \\
\hline 469-76 ou Taiwan A-144 & 5,2 & Cuba 169 & 4,1 \\
\hline $\mathrm{IC}_{(\mathrm{m}) 0,95}{ }^{3}$ & $3,7 \pm 0,14$ & $\mathrm{IC}_{(\mathrm{m}) 0,95^{3}}$ & $2,9 \pm 0,12$ \\
\hline$C I_{(m) 0.95}$ & & $C I_{(m) 0.95}$ & \\
\hline $\operatorname{CV}(\%)^{3}$ & 16,2 & $\operatorname{CV}(\%)^{3}$ & 17,2 \\
\hline
\end{tabular}

Comprimento de folhas (CF), $\mathrm{cm}$

Leaves lenght (LL), cm

\begin{tabular}{|c|c|c|c|}
\hline \multicolumn{2}{|c|}{$\begin{array}{l}\text { Estação chuvosa }{ }^{1} \\
\text { Rainy season }\end{array}$} & \multicolumn{2}{|c|}{$\begin{array}{c}\text { Estação seca }{ }^{1} \\
\text { Dry season }\end{array}$} \\
\hline Clones de menor CF & $\mathrm{CF}(\mathrm{cm})$ & Clones de menor CF & $\mathrm{CF}(\mathrm{cm})$ \\
\hline Clones with the lowest $L L$ & $L L(\mathrm{~cm})$ & Clones with the lowest $L L$ & $L L(\mathrm{~cm})$ \\
\hline CE 6 A.D & 62,2 & Kizozi & 48,5 \\
\hline Cameroon & 62,2 & Ibitinema & 56,0 \\
\hline CAC 262 & 63,7 & 415-76 ou Mercker & 58,2 \\
\hline CPAC & 65,2 & 479-76 ou Terezópolis & 59,0 \\
\hline Porto Rico - 534 - B & 65,5 & Três Rios & 59,7 \\
\hline Clones de maior CF & $\mathrm{CF}(\mathrm{cm})$ & Clones de maior CF & $\mathrm{CF}(\mathrm{cm})$ \\
\hline Clones with the highest $L L$ & $L L(\mathrm{~cm})$ & Clones with the highest $L L$ & $L L(\mathrm{~cm})$ \\
\hline 477-76(Napier/CNPGL) & 90,0 & Cuba 169 & 81,0 \\
\hline Venezuela A.D & 90,2 & Vrukuona A.D & 83,2 \\
\hline Cuba 115 & 90,5 & Mineirão/IPEACO & 83,2 \\
\hline 470-76 ou Napier SEA & 91,2 & Hexaplóide & 84,0 \\
\hline 414-76 (Elef. da Colômbia) & 91,2 & 473-76 (Elefante de Pinda) & 85,5 \\
\hline $\begin{array}{c}\mathrm{IC}_{(\mathrm{m}) 0,95}{ }^{3} \\
C I_{(m) 095}\end{array}$ & $78,2 \pm 1,74$ & $\begin{array}{c}\mathrm{IC}_{(\mathrm{m}) 0,95}{ }^{3} \\
C I_{(m) 095}\end{array}$ & $71,4 \pm 1,60$ \\
\hline $\mathrm{CV}(\%)^{3}$ & 9,6 & $\mathrm{CV}(\%)^{3}$ & 9,7 \\
\hline
\end{tabular}

1 Avaliação realizada em 1998.

2 Avaliação realizada em 1997.

$3 \mathrm{IC}_{(\mathrm{m}) 0,95}=$ intervalo de confiança para a média com um nível de confiança de $95 \%$ de probabilidade; valores estimados para os 71 clones.

3 Coeficiente de variação.

1 Evaluation performed in 1998.

2 Evaluation performed in 1997.

${ }^{3} \mathrm{Cl}_{(\mathrm{m}) 0.95}=$ Mean confidence interval (confidence level of 95\%); values obtained from 71 clones.

${ }^{3}$ Coefficient of variation. 
ao pastejo. Isto significa que ao selecionar plantas de folhas compridas, provavelmente ter-se-á, entre outros fatores, plantas com maior PLF/área, o que representa talvez a característica mais desejável em gramíneas destinadas ao pastejo.

Ângulos foliares medial e apical

Os valores médios encontrados para os dez clones superiores e inferiores foram 38,3 e 20,1 , e 34,0 e $18,9^{\circ}$, para as estações chuvosa e seca, respectivamente (Tabela 6). O clone 464-76 ou Costa Rica apresentou os maiores valores de ângulos medial e apical na estação chuvosa, com 52,3 e $14,0^{\circ}$, respectivamente. Na seca, os clones 414-76 ou Elefante da Colômbia e 483-76 ou Turrialba apresentaram os maiores valores para ângulo medial, $\operatorname{com} 38,3^{\circ}$, tendo o segundo apresentado também o maior valor para ângulo apical, com $17,3^{\circ}$.

Oliveira (1988) encontrou valores médios para ângulo foliar do terço superior de 67,71 e $74,78^{\circ}$, para primeira e segunda colheita, respectivamente. Para o terço médio, as médias foram de 60,76 e $65,16^{\circ}$. O autor encontrou correlações positivas entre altura de planta e ângulos foliares com a produção de MS, onde os clones mais produtivos apresentaram maior altura e ângulos foliares. Vale salientar que este autor utilizou como eixo de referência o plano horizontal, enquanto que neste trabalho, foi utilizado o colmo como a referência, ou seja, o plano vertical, o que torna os ângulos complementares entre si.

Produção de matéria seca (PMS) e produção de lâmina foliar (PLF)

Os resultados de PMS nas duas estações do ano e PLF na estação seca, encontram-se na Tabela 7 . A PMS apresentou valor médio de 10,2 t/ha/60dias na estação chuvosa, enquanto na seca esse valor foi de 5,0 t/ha. A média de PMS encontrada na estação seca representou em torno de 50\% da PMS média da estação chuvosa. Isto foi devido, provavelmente, às precipitações ocorridas nas duas primeiras semanas da estação seca, o que normalmente não acontece na região. Foram observadas médias de 20,0 e 9,7 t/ha/60dias para os dez clones superiores e de 4,0 e 2,2 t/ha/60dias para os dez clones inferiores, para as estações chuvosa e seca, respectivamente. Os clones 474-76 ou Mineiro, 419-76 ou Pusa Napier n.2, 418-76 ou Mercker Sta. Rita, 468-76 ou Mercker México, Porto Rico-534-B, IJ 7127 cv. Empasc 310, BAG-50, entre outros, foram materiais dos mais produtivos na estação chuvosa, obtendo, contudo, queda acentuada no período seco.
Os dados médios de PMS encontrados neste trabalho estão de acordo com os relatos de Meirelles et al. (1998), que obtiveram médias de 12,80 e 4,38 t MS/ha, respectivamente, para estações chuvosa e seca, resultados obtidos de seis cortes realizados no período de um ano. Quando comparados aos resultados obtidos por Costa et al. (1998), os dados de PMS foram inferiores apenas na estação chuvosa. Uma vez que os autores realizaram cortes com 84 dias de intervalo, ou seja, 24 dias a mais que neste trabalho, os dados vêm demonstrar o quanto o capim-elefante é susceptível à condições de baixa disponibilidade de água, visto que as plantas não elevaram sua produtividade ao longo do maior tempo de crescimento nessas condições.

Quanto aos dados de PLF, os clones Mineirão/ IPEACO, Hexaplóide e 475-76 ou Mole de Volta Grande obtiveram valores bem superiores aos demais, com 7,4; 7,3; e 6,2 t/ha/60dias (Tabela 7), respectivamente, enquanto a média geral da coleção foi de 2,7 t/ha/60dias. Os valores de PLF observados encontram-se abaixo da média relatada por Lira et al. (1998) para sete clones de capimelefante, também em Vitória de Sto. Antão/PE, que apresentaram 6,95 t/ha/473dias, possivelmente devido à diferente altura da planta no momento da avaliação.

Foi observada uma relação positiva entre a PMS e a PLF na estação seca (PMS=-0,19+1,88.PLF; $\left.\mathrm{R}^{2}=0,90 ; \mathrm{P}<0,01\right)$, indicando que as plantas com altas PMS também apresentam altos valores para PLF, que no caso da utilização do capim-elefante sob pastejo é, possivelmente, a característica mais desejável. Assim, foi adotado este critério (PLF) na seleção de dez clones de capim-elefante para serem testados sob pastejo na fase seguinte do programa de melhoramento, aproveitando ser esta característica de mais fácil avaliação e com alta determinação sobre a PMS.

É importante salientar que o coeficiente de variação neste tipo de experimento representa a variabilidade fenotípica entre os clones estudados, e não o grau de precisão do experimento. Valores de $\mathrm{CV}$ relativamente altos encontrados para alguns caracteres como por exemplo perfilhamento axilar, produção de matéria seca e de lâmina foliar, demonstram, nesse caso, maior potencial de seleção para o caracter em estudo, enquanto baixos valores de $\mathrm{CV}$ indicariam menor variabilidade entre os clones e, portanto, mais baixa possibilidade de seleção. 
Tabela 6 - Ângulos foliares medial e apical de clones de capim-elefante

Table 6 - Medial and apex portions of leaf angle of elephantgrass clones

\begin{tabular}{|c|c|c|c|}
\hline & $\begin{array}{l}\text { Âng } \\
\text { Apex }\end{array}$ & $\begin{array}{l}\text { ical (AFA), } \\
\text { angle }(A L A),{ }^{0}\end{array}$ & \\
\hline & & & \\
\hline Clones de menor AFA & $\operatorname{AFA}\left({ }^{\circ}\right)$ & Clones de menor AFA & $\operatorname{AFA}\left({ }^{\circ}\right)$ \\
\hline Clones with the lowest ALA & $A L A\left({ }^{\circ}\right)$ & Clones with the lowest ALA & $A L A\left({ }^{\circ}\right)$ \\
\hline 481-76(Duro V. Grande) & 6,7 & CE 3 A.D & 5,0 \\
\hline P-241 Piracicaba & 7,0 & CE 4 A.D & 5,0 \\
\hline CE 8 A.D & 7,3 & 481-76(Duro V. Grande) & 6,0 \\
\hline 421-76 ou Napier Goiano & 7,3 & 421-76 ou Napier Goiano & 6,3 \\
\hline Roxo de Botucatu & 7,3 & Cuba 169 & 6,3 \\
\hline Clones de maior AFA & $\operatorname{AFA}\left({ }^{\circ}\right)$ & Clones de maior AFA & $\operatorname{AFA}\left(^{\circ}\right)$ \\
\hline Clones with the highest ALA & $A L A\left(^{\circ}\right)$ & Clones with the highest ALA & $A L A\left(^{\circ}\right)$ \\
\hline Vrukuona A.D & 13,0 & 470-76 ou Napier SEA & 14,0 \\
\hline 464-76 ou Costa Rica & 14,0 & 414-76 (Elef. da Colômbia) & 14,0 \\
\hline CE 2 A.D & 14,3 & 591-76 ou Cameroon & 14,3 \\
\hline CE 3 A.D & 16,3 & 454-76 ou Taiwan A-25 & 14,7 \\
\hline Hexaplóide & 19,3 & 483-76 ou Turrialba & 17,3 \\
\hline $\mathrm{IC}_{(\mathrm{m}) 0,95}{ }^{3}$ & $10,4 \pm 0,49$ & $\mathrm{IC}_{(\mathrm{m}) 0,95}{ }^{3}$ & $10,2 \pm 0,56$ \\
\hline$C I_{(m) 0.95}$ & & $C I_{(m) 0.95}$ & \\
\hline $\mathrm{CV}(\%)^{4}$ & 20,2 & $\mathrm{CV}(\%)^{4}$ & 23,5 \\
\hline & $\begin{array}{r}\text { Ângl } \\
\text { Medial } \\
\end{array}$ & $\begin{array}{l}\operatorname{dial}(\mathrm{AFM}),{ }^{0} \\
\text { f angle }(M L A),{ }^{0}\end{array}$ & \\
\hline & $\mathrm{osa}^{1}$ & & \\
\hline & & & \\
\hline Clones de menor AFM & $A F M\left({ }^{\circ}\right)$ & Clones de menor AFM & $A F M\left(^{\circ}\right)$ \\
\hline Clones with the lowest MLA & $M L A\left({ }^{\circ}\right)$ & Clones with the lowest MLA & $M L A\left({ }^{\circ}\right)$ \\
\hline Vrukwona & 17,7 & CE 6 A.D & 16,3 \\
\hline CE 6 A.D & 19,3 & CE 4 A.D & 16,7 \\
\hline 452-76 ou Taiwan A-148 & 19,7 & CE 9 A.D & 18,3 \\
\hline 469-76 ou Taiwan A-144 & 20,0 & 481-76(Duro V. Grande) & 18,3 \\
\hline 591-76 ou Cameroon & 20,0 & Guaçu/IZ & 19,3 \\
\hline Clones de maior AFM & $\operatorname{AFM}\left({ }^{\circ}\right)$ & Clones de maior AFM & $\operatorname{AFM}\left({ }^{\circ}\right)$ \\
\hline Clones with the highest $M L A$ & $M L A\left(^{\circ}\right)$ & Clones with the highest $M L A$ & $M L A\left(^{\circ}\right)$ \\
\hline CPAC & 38,3 & 468-76 ou Mercker México & 33,3 \\
\hline Kizozi & 38,3 & Gigante de pinda & 33,3 \\
\hline 483-76 ou Turrialba & 39,3 & Porto Rico - 534 - B & 33,3 \\
\hline 468-76(Mercker México) & 40,0 & 483-76 ou Turrialba & 38,3 \\
\hline 464-76 ou Costa Rica & 52,3 & 414-76 (Elef. da Colômbia) & 38,3 \\
\hline $\mathrm{IC}_{(\mathrm{m}) 0,95}{ }^{3}$ & $28,8 \pm 1,42$ & $\mathrm{IC}_{(\mathrm{m}) 0,95}{ }^{3}$ & $25,7 \pm 1,12$ \\
\hline $\mathrm{CI}_{(\mathrm{m}) 0.95}$ & & $\mathrm{CI}_{(\mathrm{m}) 0.95}$ & \\
\hline $\mathrm{CV}(\%)^{4}$ & 21,2 & $\mathrm{CV}(\%)^{4}$ & 18,7 \\
\hline $\begin{array}{l}1 \text { Avaliação realizada em } 1998 . \\
2 \text { Avaliação realizada em } 1997 . \\
3{ }^{1} \mathrm{C}_{(\mathrm{m}) 0,95}=\text { intervalo de confiança } \\
{ }^{4} \text { Coeficiente de variação. } \\
{ }^{1} \text { Evaluation performed in } 1998 . \\
{ }^{2} \text { Evaluation performed in } 1997 . \\
{ }^{3} \mathrm{Cl}_{(m) 0.95}=\text { mean confidence interval }\end{array}$ & com um níve & 95\% de probabilidade; valor & os 71 clones. \\
\hline & & & \\
\hline
\end{tabular}


Tabela 7 - Produção de matéria seca e de lâmina foliar de clones de capim-elefante

Table 7 - Dry matter and leaf blade yield of elephantgrass clones

\begin{tabular}{|c|c|c|c|}
\hline \multicolumn{4}{|c|}{$\begin{array}{c}\text { Produção de matéria seca (PMS), } \mathrm{t} \text { de MS/ha/corte } \\
\text { Dry matter yield (DMY), tDM/ha/cut }\end{array}$} \\
\hline \multicolumn{2}{|c|}{$\begin{array}{l}\text { Estação chuvosa } \\
\text { Rainy season }\end{array}$} & \multicolumn{2}{|c|}{$\begin{array}{l}\text { Estação seca } \\
\text { Dry season }\end{array}$} \\
\hline Clones de menor PMS & PMS & Clones de menor PMS & PMS \\
\hline Clones with the lowest DMY & $D M Y$ & Clones with the lowest DMY & $D M Y$ \\
\hline CAC 262 & 2,3 & IJ-7141 & 0,9 \\
\hline CE 8 A.D & 2,5 & Três Rios & 1,3 \\
\hline CE 6 A.D & 3,6 & CPAC & 1,8 \\
\hline Roxo de Botucatu & 3,7 & 464-76 ou Costa Rica & 2,3 \\
\hline 464-76 ou Costa Rica & 3,9 & 419-76 ou Pusa Napier n.2 & 2,5 \\
\hline Clones de maior PMS & PMS & Clones de maior PMS & PMS \\
\hline Clones with the highest DMY & $D M Y$ & Clones with the highest DMY & $D M Y$ \\
\hline Napiezinho & 17,8 & BAG-50 & 8,0 \\
\hline Gigante de pinda & 19,0 & King Grass & 10,7 \\
\hline 468-76(Mercker México) & 20,0 & 475-76 (Mole V. Grande) & 11,7 \\
\hline IJ-7127 cv. Empasc 310 & 25,7 & Hexaplóide & 12,9 \\
\hline BAG- 50 & 30,9 & Mineirão/IPEACO & 15,6 \\
\hline $\mathrm{IC}_{(\mathrm{m}) 0,95}{ }^{3}$ & $10,2 \pm 1,28$ & $\mathrm{IC}_{(\mathrm{m}) 0,95}{ }^{3}$ & $5,0 \pm 0,58$ \\
\hline $\mathrm{CI}_{(\mathrm{m}) 0.95}$ & & $\mathrm{CI}_{(\mathrm{m}) 0.95}$ & \\
\hline $\mathrm{CV}(\%)^{4}$ & 53,9 & $\mathrm{CV}(\%)^{4}$ & 50,0 \\
\hline
\end{tabular}

Produção de lâminas foliares (PLF), t de MS/ha/corte

Leaf blade yield (LBY), $t$ DM/ha/cut

\begin{tabular}{cccc}
\hline & \multicolumn{3}{c}{$\begin{array}{c}\text { Estação seca } \\
\text { Dry season }\end{array}$} \\
\hline Clones de menor PLF & PLF & Clones de maior PLF & PLF \\
Clones with the lowest LBY & LBY & Clones with the highest LBY & LBY \\
\hline IJ-7141 & 0,6 & 591-76 ou Cameroon & 4,8 \\
Três Rios & 1,0 & King Grass & 5,1 \\
CPAC & 1,1 & 475-76 (Mole V. Grande) & 6,2 \\
CE 6 A.D & 1,3 & Hexaplóide & 7,3 \\
419-76 ou Pusa Napier n.2 & 1,3 & Mineirão/IPEACO & 7,4 \\
IC $_{(\mathrm{m}) 0,95}$ & & $2,7 \pm 0,30$ & \\
CI $_{(\mathrm{m}) 0.95}$ & & & \\
CV $(\%)^{4}$ & & 48,1 & \\
\hline
\end{tabular}

1 Avaliação realizada em 1998.

2 Avaliação realizada em 1997.

$3 \mathrm{IC}_{(\mathrm{m}) 0,95}=$ intervalo de confiança para a média com um nível de confiança de $95 \%$ de probabilidade; valores estimados para os 71 clones.

4 Coeficiente de variação.

1 Evaluation performed in 1998.

2 Evaluation performed in 1997.

${ }^{3} \mathrm{Cl}_{(m) 0.95}=$ mean confidence interval (confidence level of 95\%); values obtained from 71 clones.

${ }^{4}$ Coefficient of variation.

\section{Conclusões}

Os clones com maior produção de matéria seca, nas duas estações do ano, em geral, apresentaram maiores alturas de planta e de meristemas apicais. Por outro lado, na estação seca, os clones mais produtivos apresentaram maiores diâmetros de colmo, relação colmo/folha, número de perfilhos basais e total e comprimento de folhas.

Excetuando-se a relação $\mathrm{C} / \mathrm{F}$, os clones mais produtivos quanto à produção de lâmina foliar, tam- bém relacionaram-se positivamente com as características anteriormente citadas, na estação seca.

Os materiais Hexaplóide e Mineirão/IPEACO destacaram-se por apresentarem altas produções de matéria seca e de lâmina foliar na estação seca.

Os clones mais promissores a serem testados sob pastejo foram o Mineirão/IPEACO, Hexaplóide, Mole de Volta Grande, King Grass, 591-76 ou Cameroon, CE 5 A.D., Gigante de Pinda, CE 4 A.D., Elefante da Colômbia e Vrukwona. 


\section{Literatura Citada}

ANUÁRIO DA PECUÁRIA BRASILEIRA. São Paulo: Argos Comunicação, 1998. 385p.

ANUÁRIO ESTATÍSTICO DE PERNAMBUCO. Recife: IBGE, 1991. v.40.

BOTREL, M.A.; ALVIM, M. J.; MARTINS, C.E. Avaliação e seleção de cultivares de capim-elefante (Pennisetum purpureum, Schum) para pastejo. Revista da Sociedade Brasileira de Zootecnia, v.23, n.5, p.754-762, 1994.

BOTREL, M.A.; PEREIRA, A.V.; XAVIER, D.F. et al. Avaliação de novos clones de capim-elefante, para utilização sob pastejo. In: REUNIÃO ANUAL DA SOCIEDADE BRASILEIRA DE ZOOTECNIA, 35., 1998, Botucatu. Anais... Botucatu: Sociedade Brasileira de Zootecnia, 1998. p.489-491.

COSTA, N.L.; TOWNSEND, C.R.; MAGALHÃES, J.A. et al. Avaliação agronômica de clones de capim elefante (Pennisetum purpureum, Schum.) em Rondônia. In: REUNIÃO ANUAL DA SOCIEDADE BRASILEIRA DE ZOOTECNIA, 35., 1998, Botucatu. Anais... Botucatu: Sociedade Brasileira de Zootecnia, 1998. p.339-341.

DELVIN, R.M. Fisiología vegetal. Barcelona: Omega, 1997. $468 \mathrm{p}$.

ENCARNAÇÃO, C.R.F. Observações meteorológicas e tipos climáticos das unidades e campos experimentais da Empresa IPA. Recife: IPA, [1980].

GOMIDE, C.A.M. Morfogênese e análise de crescimento de cultivares de Panicum maximum (Jacq.). Viçosa, MG: Universidade Federal de Viçosa, 1997. 53p. Dissertação (Mestrado em Zootecnia) - Universidade Federal de Viçosa, 1997.

GONÇALVES, C.A.; SIMÃO NETO, M.; VEIGA, J.B. 1979. Comparação de cultivares e híbridos de capim-elefante. Pesquisa Agropecuária Brasileira, v.14, n.4, p.359-364.

EMPRESA PERNAMBUCANA DE PESQUISA AGROPECUÁRIA - IPA (Recife, PE). Bancos de dados metereológicos. Recife, 1994. 137p.
LIRA, M.A.; DUBEUX Jr., J.C.B.; OLIVEIRA, C.F. et al. Competição de cultivares de capim-elefante (Pennisetum purpureum, Schum) e de seus híbridos com milheto (P. americanum (L.) Leeke), sob pastejo. In: REUNIÃO ANUAL DA SOCIEDADE BRASILEIRA DE ZOOTECNIA, 35., 1998, Botucatu. Anais... Botucatu: Sociedade Brasileira de Zootecnia, 1998. p.421-423.

MEIRELlES, P.R.L.; MOCHIUTTI, S.; PEREIRA, A.V. Avaliação de genótipos de capim elefante (Pennisetum purpureum, Schum.) em área de várzea alta no Amapá. In: REUNIÃO ANUAL DA SOCIEDADE BRASILEIRA DE ZOOTECNIA, 35., 1998, Botucatu. Anais... Botucatu: Sociedade Brasileira de Zootecnia, 1998. p.632-634.

OLIVEIRA, J.A.C. Potencial forrageiro de clones de capimelefante (Pennisetum purpureum, Schum) e de capimelefante $\mathbf{x}$ milheto (Pennisetum americanum (L) Leeke) no agreste semi-árido de Pernambuco: Recife, PE: Universidade Federal Rural de Pernambuco, 1988. 233p. Dissertação (Mestrado em Produção Animal) - Universidade Federal Rural de Pernambuco, 1988.

SANTOS, M. do C.M. dos; TABOSA, J.N.; DIAS, F.M. et al. Comportamento de clones de capim-elefante e de híbridos de capim-elefante $\mathrm{x}$ milheto no Semi-Árido do Nordeste do Brasil. Pesquisa Agropecuária Brasileira, v.29, n.10, p.1609-1615, 1994.

THORNTHWAITE, C.W.; MATTER, J.R. The water oudget and its use irrigation. In: THORNITHWAITE, C.W.; MATTER, J.R. (Eds.) Water the year book of agriculture. Washington: USDA, 1955. p.356-358.

XAVIER, D.F.; BOTREL, M.A.; DAHER, R.F. et al. Caracterização morfológica e agronômica de algumas cultivares de capim-elefante. Coronel Pacheco: EMBRAPA-CNPGL, 1955. 24p. (EMBRAPA-CNPGL, Documentos, 60).

Recebido em: 12/02/01

Aceito em: 19/07/01 